Original scientific paper

\title{
STABILITY OF A FACE GUARD IN A LARGE MINING HEIGHT WORKING FACE
}

\author{
Wang, D. L. ${ }^{* * *}$; Zeng, X. T. *\#; Wang, G. F. ${ }^{* * * * * * *} \&$ Li, R. ${ }^{* * *}$ \\ ${ }^{*}$ School of Mechanical Electronic and Information Engineering, China University of Mining and \\ Technology Beijing, Beijing, 100083, China \\ ** Beijing Tianma Electronic-Hydraulic Control System Company Ltd., Beijing, 100013, China \\ ${ }^{* * * *}$ China Coal Technology and Engineering Group Corp, Beijing, 100013, China \\ Coal Mining Research Institute, China Coal Technology and Engineering Group, Beijing, 100013, \\ China \\ E-Mail: zengxt@ @umtb.edu.cn ( ${ }^{\#}$ Corresponding author)
}

\begin{abstract}
Hydraulic support is an important piece of equipment in a fully mechanized mining face, and it is used to prevent the roof from falling out of control and protect the workers underground. The stability of hydraulic support under different conditions is related to security and must be taken seriously. To analyse the mechanical response of hydraulic support, a rigid-flexible coupling numerical analysis model of the face guard mechanism, which is part of hydraulic support, is established based on the multi-body dynamics software ADAMS. The equivalent spring is used to replace the face sprag ram to analyse the influence of the structural types of face guards on the stability of a coal wall under various coupling conditions with a coal wall. The support characteristics of the face guard mechanism are also explored. Results show that the errors of the contact characteristics of the face guard mechanism between the theoretical and numerical calculations are less than $3 \%$. Compared with the split face guard, the integral face guard exhibits greater flexibility and more favourable stability under different coupling states. The study provides a reference for optimizing face guards with hydraulic support. (Received in May 2021, accepted in August 2021. This paper was with the authors 1 month for 1 revision.)
\end{abstract}

Key Words: Coal Wall Spalling, Hydraulic Support, Face Guard Mechanism, Coupling Relationship

\section{INTRODUCTION}

Coal is an important resource, and as one of the largest coal-consuming countries in the world, China's coal consumption accounts for $51.7 \%$ worldwide [1]. As an important piece of equipment used in coal mining, hydraulic support provides essential guarantee to a safe and efficient mining environment. With the rapid advancement of mining technology and equipment and the gradual improvement of hydraulic support and surrounding rock coupling theory in recent years $[2,3]$, fully mechanized mining equipment with a large mining height, represented by hydraulic supports with a large mining height, has been widely used. However, production practice and theoretical analysis have demonstrated that coal wall spalling is affected by many factors, such as in situ stress distribution characteristics, coal strength, and supporting resistance $[4,5]$. Among these factors, an increase in mining height weakens the stability of a coal wall with increasing frequency and depth of spalling, causing roof flaking [6]. This phenomenon leads to the further deterioration of mining conditions, restricting mining efficiency and may eventually trigger safety accidents [7].

A reliable and stable hydraulic support for a large mining height is significant for reducing safety accidents and protecting underground workers. Therefore, the support capacity and mechanical response of hydraulic support under different support conditions are essential research topics. To analyse the steady and dynamic effects of different types of face guard mechanism on coal wall, a multi-rigid-body numerical model of a face guard plate is established on the basis of the multi-body dynamics software ADAMS. The rigid-flexible 
coupling analysis model is established through the flexible replacement of each structural component of the face guard plate to compare the contact behaviour and overall stress characteristics of the two types of face guard and coal wall under different contact states. The findings of this study provide a reference for the structural design of face guards with hydraulic supports.

\section{STATE OF THE ART}

Hydraulic support is considered the key supporting equipment in a working face, and its support mechanism provides a horizontal supporting force to coal walls. Pang et al. [8] pointed out that the failure and instability of coal walls are affected by the properties of the coal bed, mining height, and other factors. Liu [9] analysed the control effect of hydraulic support and reported that the horizontal support force on a coal wall can improve its stability. Ge et al. [10] established a model of hydraulic support and the environmental surface; they indicated that the simulation method is feasible for simulating the adaptive process. Meanwhile, the simulation method has been proven effective for technology optimization in different fields [11]. Hu [12] proposed a cooperative double closed-loop control technology to ensure secure contact between the roof and the canopy; they determined that canopy posture can be controlled well by using the proposed method. Behera et al. [13] explored the stress distribution, failure characteristics, and horizontal displacement of a coal wall. They tested the critical value of stress when horizontal displacement occurred and found that the spalling of the coal wall probably occurs in the upper middle position of the working face. Verma and Deb [14] established a coupling model of hydraulic support and longwall panels by using 2D continuum finite element models; they identified depth as the most significant major effect in statistical models. Zeng et al. [15] established a hydraulic support model for obtaining pose and stress state variations under impact load. They determined that the force at the hinge point between the roof beam and the shield beam was the strongest. Witek and Prusek [16] analysed the bearing state of hydraulic support under different sizes and positions of applied external load by using the finite element method; the results of the numerical analyses were highly consistent with those of the laboratory tests. Liang et al. [17] explored the relationship between mechanical-hydraulic coupling rigidity and the parameters of a hydraulic cylinder. Islavath et al. [18] analysed the dynamic behaviour of the canopy and the hydraulic leg under different mining parameters by using the finite element method. Liu et al. [19] explored the mechanical characteristics of the face guard mechanism via numerical simulation and found that the face guard mechanism and ram exhibited different characteristics under varying roof pressures.

Through the aforementioned studies, the face guard mechanism is determined to be conducive to maintaining the stability of a coal wall. To date, however, only a few studies have investigated the support mechanism of a face guard. Many references have simply assumed that the face guard plate is a rigid body and developed a hypothesis on the ideal coupling state between a safe guard plate and a coal wall. In an actual working face, the coal wall is no longer flat after the shearer cuts the coal, resulting in varying contact behaviour of the face guard against the coal wall. Therefore, contact characteristics should be analysed in many cases. Apart from the static maintenance function, dynamic disturbance to the coal wall should also be considered. Therefore, theoretical and numerical simulation methods have been adopted to study the dynamic response of hydraulic support under different contact characteristics during coal mining to provide references for designing hydraulic supports.

The reminder of this study is organized as follows. Section 3 introduces the primary structure type of the face guard mechanism in field mining and the different contact characteristics of hydraulic support and coal wall. Section 4 simulates the working process of 
the face guard mechanism and presents a comparative analysis of the mechanical characteristics of the face guard mechanism under different coal mining conditions. Section 5 summarizes the conclusions drawn from the study.

\section{METHODOLOGY}

\subsection{Two face guard mechanisms with hydraulic support}

At present, two major structural forms of hydraulic support are used in a large mining height working face: (1) the integral type and (2) the split type. In accordance with relevant parameters, numerical models are established using Creo software, as shown in Fig. 1. In this figure, No. 1 is the top beam, No. 2 is the telescopic beam, No. 3 is the first-level face guard, No. 4 is the second-level face guard, No. 5 is the third-level face guard, No. 6 is the telescopic beam jack, No. 7 is the first-level face guard jack, No. 8 is the second-level face guard jack, No. 9 is the third-level face guard jack, No. 10 is connecting rod A, and No. 11 is connecting rod B. The function and composition of the two types of face guard are basically similar. However, in the integral face guard structure with hydraulic support, four connecting rods are utilized to connect the first-level face guard with the telescopic beam. By contrast, the split type is connected by a simple hinge joint between the first-level face guard and the top beam.

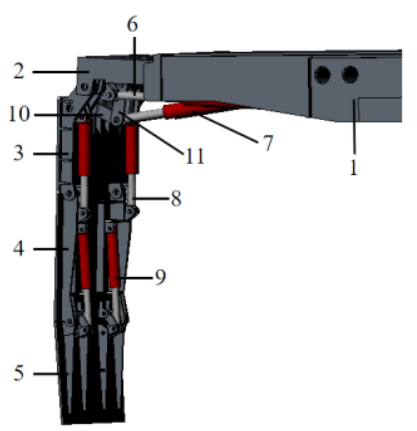

a) Numerical model of an integral face guard

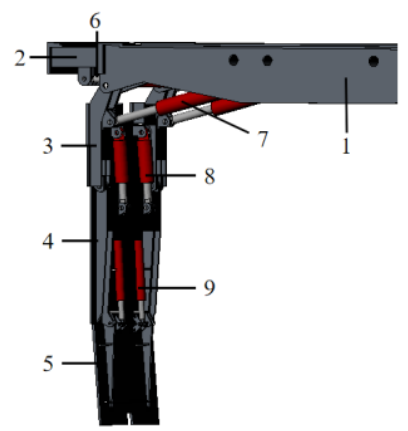

b) Numerical model of a split face guard

Figure 1: 3D models of the face guard mechanism.

\subsection{Numerical model of the face guard mechanism by using ADAMS software}

Importing the 3D models of the two types of face guard into ADAMS software is conducive to establishing a multi-rigid-body dynamic analysis model, as shown in Fig. 2.

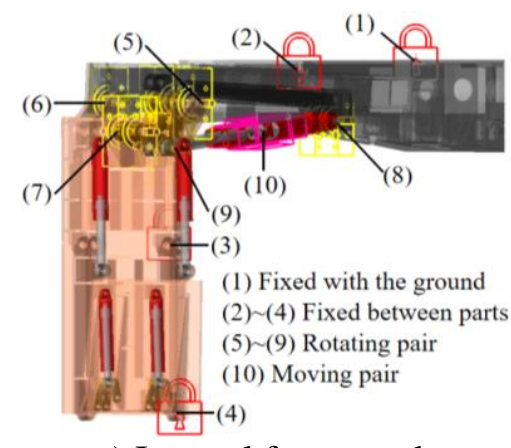

a) Integral face guard

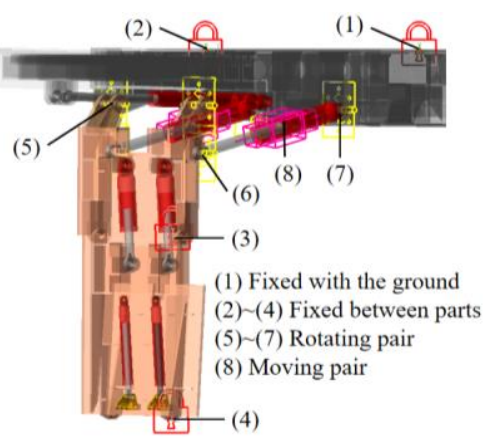

b) Split face guard

Figure 2: Rigid body numerical analysis models of the face guard mechanism.

Defining the connection between integral and split face guards is basically the same with each structural member being considered a rigid body. The connection among the telescopic beam, face guard, face guard jack, and other components is defined as a rotating pair. The 
first-level face guard jack is defined as a moving pair, and the different levels of face guard are defined as a fixed connection [20]. A driving force is applied to the rotating pair of the first-level face guard jack to control the extension speed of the two types of first-level face guard jack to reach $100 \mathrm{~mm} / \mathrm{s}$. Then, the results are analysed.

The friction coefficient of each connection is set to 0.3. The top beam, the shield beam, and other parts are supposed to be fixed to the ground to keep them static. All the levels of the face guard jacks are supposed to maintain a consistent model with the specific parameters of all the levels provided in Table I.

Table I: Parameters of all the levels of face guard jacks.

\begin{tabular}{|l|c|c|c|}
\hline \multicolumn{1}{|c|}{ Item } & $\begin{array}{c}\text { Cylinder diameter } \\
(\mathrm{mm})\end{array}$ & $\begin{array}{c}\text { Rod diameter } \\
(\mathrm{mm})\end{array}$ & $\begin{array}{c}\text { Effective resistance } \\
(\mathrm{kN})\end{array}$ \\
\hline First-level face guard jack & 125 & 90 & 490 \\
\hline Second-level face guard jack & 100 & 70 & 300 \\
\hline Third-level face guard jack & 80 & 60 & 190 \\
\hline
\end{tabular}

\subsection{Theoretical analysis of the contact characteristics between the integral face guard and the coal wall}

The stress diagram of an integral face guard is shown in Fig. 3, wherein the coordinate system is established by setting the hinge point of the telescopic beam and the first-class retaining plate as the origin $\mathrm{O} . \mathrm{OA}, \mathrm{OC}, \mathrm{AB}$, and $\mathrm{BC}$ constitute a four-bar linkage mechanism, in which $F_{1}$ is the force of the first-level face guard jack on hinge point $\mathrm{B}, F_{2}$ is the force of the secondlevel face guard jack on hinge point $\mathrm{E}$, and $F_{i j}$ is the force of point $i$ on point $j$. The distance from point $\mathrm{O}$ to $\operatorname{rod} \mathrm{BC}$ is $L$, and the distance from point $\mathrm{O}$ to $\operatorname{rod} \mathrm{AB}$ is $N$. The distance of the extension line from point $\mathrm{O}$ to $F_{1}$ is $M$, and the distance of the extension line from point $\mathrm{D}$ to $F_{2}$ is $K . y_{P}$ is the vertical distance from the external load $P$ to the corresponding hinge point $\mathrm{O}$. $\theta$ is determined as the angle between the force and the horizontal direction of the first-level face guard jack, and $\alpha$ is fixed as the angle between the bar BC and the horizontal direction. On the basis of the preceding information, the mathematical model of the contact characteristics of the integral face guard is expressed as shown in Eq. (1).

$$
\left\{\begin{array}{l}
F_{1} \cdot \cos \theta-F_{B C} \cdot \cos \alpha=0 \\
F_{1} \cdot \sin \theta-F_{B C} \cdot \sin \alpha=0 \\
F_{A B} \cdot N=F_{B C} \cdot L \\
F_{A B} \cdot N=F_{1} \cdot M \\
F_{B C} \cdot L=P \cdot y_{p}
\end{array}\right.
$$

When the external load is in the first-level face guard, the value $P_{1}$ can be obtained. When the external load $P$ is in the second-level face guard, the maximum working resistance of the second-level face guard jack must be taken into account and it is supplemented by Eq. (2).

$$
\left\{\begin{array}{cc}
P=P_{1} & P_{1} \cdot\left(y_{D}-y_{P}\right)-F_{2} \cdot K \leq 0 \\
P \cdot\left(y_{D}-y_{P}\right)=F_{2} \cdot K & P_{1} \cdot\left(y_{D}-y_{P}\right)-F_{2} \cdot K>0
\end{array}\right.
$$

According to the model size parameters of integral face guard, some corresponding coefficients are determined: $M=342.4 \mathrm{~mm}, N=136.43 \mathrm{~mm}, K=111.95 \mathrm{~mm}$. The loading position of the external load $P$ determines $100 \mathrm{~mm}<y_{P}<2100 \mathrm{~mm}$, whereas $y_{D}=-1049.75$ mm, including the angle $\alpha=24^{\circ}$.

In accordance with relevant parameters, the analysis results of the contact characteristics of the integral face guard are shown in Fig. 4. When the distance from the contact position to 
the hinge point $\mathrm{O}$ of the face guard and the coal wall is less than $300 \mathrm{~mm}$, the first-level face guard jack is in tension state. With the downward movement of the contact position between the coal wall and the face guard (regarded as the form of point contact), the first-level face guard jack is compressed. Thus, bearing capacity decreases gradually, with the maximum bearing capacity reaching $922 \mathrm{kN}$. Under the secondary retaining plate, bearing capacity is only $64 \mathrm{kN}$, indicating that the face guard force provided by the retaining plate to the coal wall is relatively small.

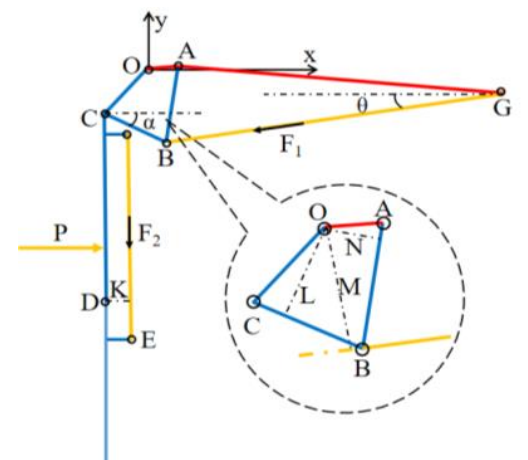

Figure 3: Stress analysis diagram of the integral face guard.

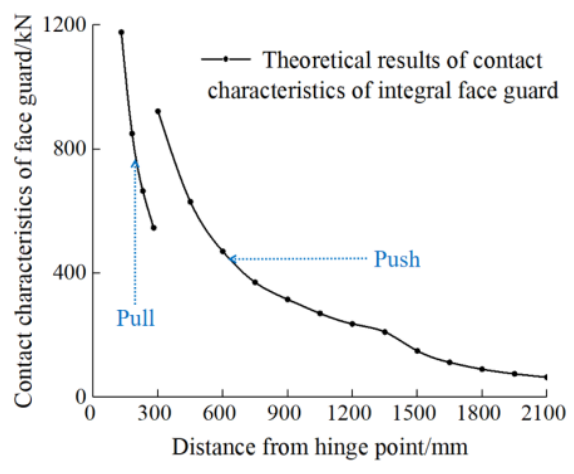

Figure 4: Theoretical values of the contact characteristics of the integral face guard.

\subsection{Theoretical analysis of the contact characteristics between the split face guard and the coal wall}

Fig. 5 shows the stress analysis of a split face guard. The first-level face guard and the top beam are simply hinged together. The ultimate support force at different positions of the midline can be obtained through the moment equation. A coordinate system is established with the hinge point of the first-level face guard and the top beam as the coordinate origin $\mathrm{O}$. $F_{1}$ denotes the force of the first-level face guard jack on point D, while $F_{2}$ denotes the force of the second-level face guard jack on point B. The first-level face guard is not in contact with the coal wall during the working process; thus, we must only consider the external load at the second-level face guard and the maximum working resistance of the first- and second-level face guard jacks.

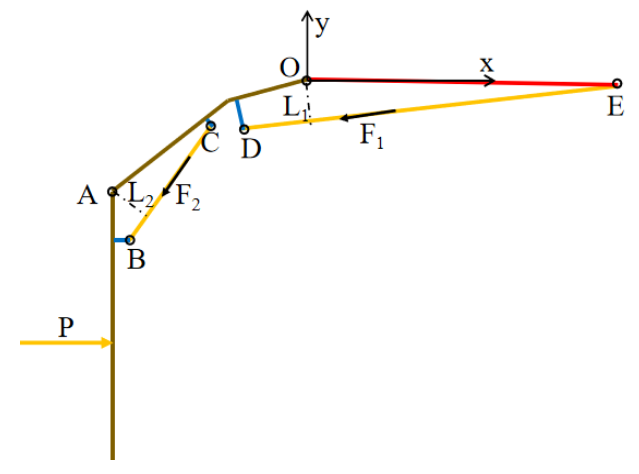

Figure 5: Stress analysis diagram of the split face guard.

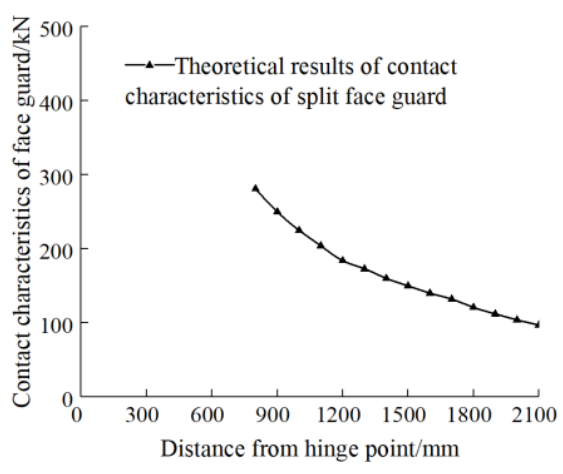

Figure 6: Theoretical value of the contact characteristics of the split face guard.

When only the maximum working resistance of the first-level face guard jack is considered, the calculation formula is expressed as Eq. (3).

$$
F_{1} \cdot L_{1}=P_{1} \cdot y
$$

When the stress of the second-level face guard jack is considered, it can be supplemented by Eq. (4). 


$$
\left\{\begin{array}{cc}
P=P_{1} & P_{1} \cdot\left(y_{A}-y_{P}\right)-F_{2} \cdot L_{2} \leq 0 \\
P \cdot\left(y_{A}-y_{P}\right)=F_{2} \cdot L_{2} & P_{1} \cdot\left(y_{A}-y_{P}\right)-F_{2} \cdot L_{2}>0
\end{array}\right.
$$

According to the model parameters of the split face guard, $L_{1}=229.65 \mathrm{~mm}$, $L_{2}=239.95 \mathrm{~mm}, y_{A}=-615.03 \mathrm{~mm}, 800 \mathrm{~mm}<y_{P}<2100 \mathrm{~mm}$. To ensure the uniqueness of the variables, two jacks with a maximum working resistance of $490 \mathrm{kN}$ are used for the first-level face guard, while two jacks with a maximum working resistance of $300 \mathrm{kN}$ are used for the second-level face guard. Only the pressure state of the jack should be considered in the split face guard due to its structural characteristics. Therefore, $F_{1}=980 \mathrm{kN}$ and $F_{2}=600 \mathrm{kN}$. As shown in Fig. 6, the change trend of the contact characteristics of the split face guard is similar to that of the integral face guard. As the contact point between the coal wall and the face guard moves downward, the effective supporting force provided by the face guard to the coal wall gradually decreases. The maximum value is approximately $283 \mathrm{kN}$, whereas the minimum value is $96 \mathrm{kN}$.

\section{RESULTS AND DISCUSSIONS}

\subsection{Numerical simulation of the contact characteristics between the face guard and the coal wall}

Parts of the components of the face guard are meshed using HyperMesh software, and a rigid connection area is established at the hinge point [21], as shown in Fig. 7. Then, the components of the rigid body's numerical model in Fig. 2 are replaced, and a rigid-flexible coupling numerical analysis model (the top beam, shield beam, and other parts are maintained as rigid bodies) is established. The telescopic beam and the face guard, connecting rod A and the face guard, connecting rod B and the telescopic beam, and two connecting rods are connected by rotating pairs. The friction coefficient is defined as 0.3 . The rigid body components, such as the top beam and the cover beam, are fixed and connected to the ground to keep them stationary.

In the numerical simulation, the face guard jacks at all the levels are replaced with the equivalent spring. The variable stiffness equivalent spring is defined in accordance with Eq. (5) of the equivalent stiffness of the hydraulic cylinder.

$$
k=\frac{A \eta}{L_{y}}
$$

where, $k$ is the stiffness coefficient of the equivalent spring $(\mathrm{N} / \mathrm{m}), A$ is the effective pressure area in the hydraulic cylinder $\left(\mathrm{m}^{2}\right), \eta$ is the bulk modulus of the hydraulic fluid (set as $1950 \mathrm{MPa}$ ), and $L_{y}$ is the length of the effective liquid column in the hydraulic cylinder (m). $L_{y}$ varies with the working height of the support.

On the basis of the aforementioned numerical model, points with an interval of $260 \mathrm{~mm}$ at the vertical centreline positions of the integral and split face guards are selected and exerted with different loads to simulate the contact characteristics of the two face guards. Load amplitude is estimated using the preceding theoretical analysis results and corrected numerous times in accordance with the response of the face guard jack. Finally, the ultimate loadbearing capacity of each position is recorded and a curve is drawn.

The comparison results are shown in Fig. 8. The maximum error between the theoretical calculation and numerical analysis results is less than $3 \%$, demonstrating the validity and accuracy of the two models. Setting $1500 \mathrm{~mm}$ from the hinge point as the dividing line, i.e., from $0 \mathrm{~mm}$ to $1500 \mathrm{~mm}$, the supporting capacity of the integral face guard is significantly better than that of the split face guard. Beyond $1500 \mathrm{~mm}$, however, the supporting capacity of the integral face guard is slightly lower than that of the split face guard; nevertheless, the 
difference is inevident. In summary, the contact characteristic of the integral face guard is better than that of the split face guard at the centreline of the face guards.

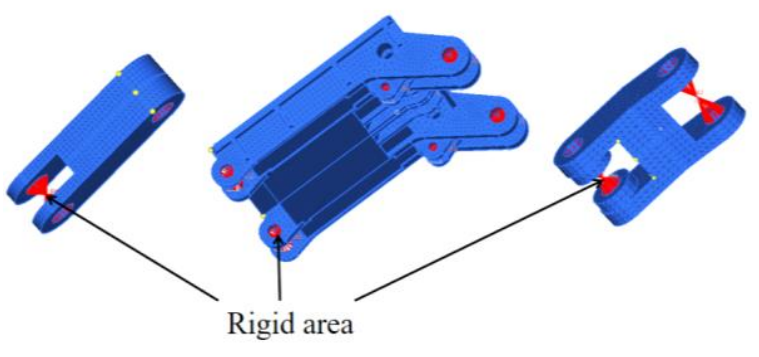

Figure 7: Part of the flexible components.

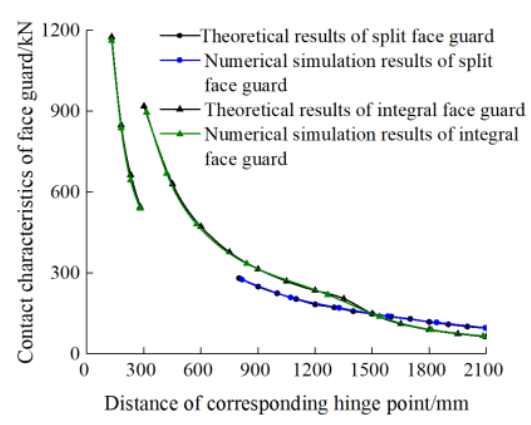

Figure 8: Comparison of the theoretical analysis and numerical simulation results.

After the coal shearer cuts the coal, many types of coupling state occur between the hydraulic support of the face guard and the coal wall. In such case, simply regarding the loading position of the external load as the centreline of the face guard does not reflect the actual situation. Therefore, the preceding analysis should be improved further. Accordingly, on the basis of the aforementioned numerical analysis model, point loads are applied to different positions of the face guard, as shown in Fig. 9. Using the integral face guard as an example, the centreline of the circular hole at the hinge of the first-level face guard and the telescopic beam is set as the X-axis (in the split face guard, the centreline of the circular hole at the hinge of the face guard and the top beam is set as the $\mathrm{X}$-axis). The centreline perpendicular to the $\mathrm{X}$-axis is used as the $\mathrm{Y}$-axis, and the loading point is set at every interval $\Delta L=275 \mathrm{~mm}$ in the $\mathrm{X}$-axis direction and every interval $\Delta M=264 \mathrm{~mm}$ in the $\mathrm{Y}$-axis direction. To facilitate the comparative analysis of the integral and split face guards, the first-level integral face guard jack only considers the compression state; hence, the loading position is $y_{P}>300 \mathrm{~mm}$. The first- and second-levels integral face guard and the second-level split face guard adopt this loading method to simulate the different contact modes of the coal wall and the face guard.

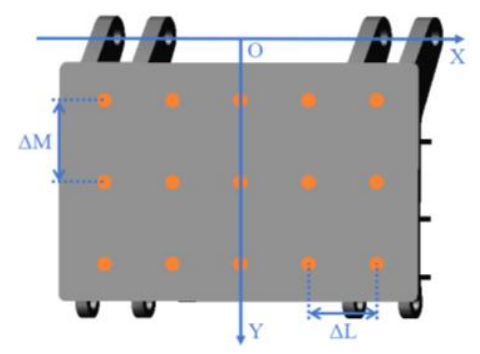

Figure 9: Diagram of loading position.

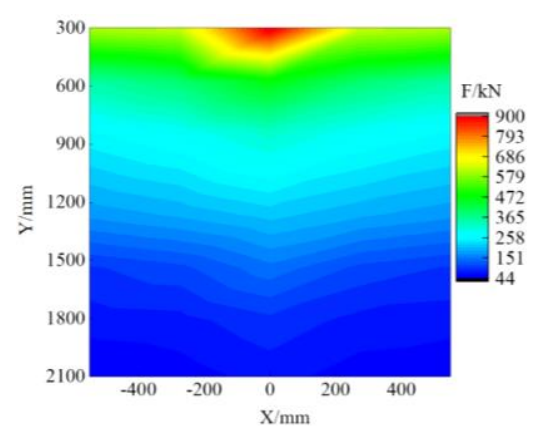

a) Integral face guard

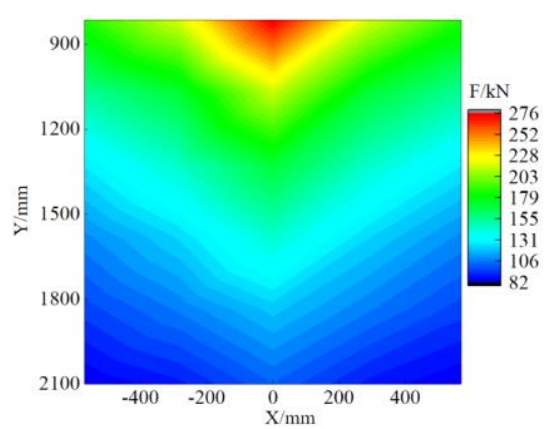

b) Split face guard

Figure 10: Numerical analysis results of the contact characteristics of the face guard. 
Fig. 10 shows the simulation analysis results of the contact characteristics of the integral and split face guards. In accordance with the data in Fig. 10, the maximum supporting capacity of the integral face guard is $900 \mathrm{kN}$ and the minimum is $47 \mathrm{kN}$. The maximum supporting capacity of the split face guard is $276 \mathrm{kN}$ and the minimum is $82 \mathrm{kN}$. In summary, the integral face guard has a better supporting range and supporting capacity than the split face guard, with better contact characteristics, under different contact conditions. Therefore, the integral face guard can better support a coal wall.

\subsection{Influence of coal wall on the mechanical characteristics of the two face guard mechanisms}

To make a comparative analysis on the mechanical response characteristics of the two structural forms of face guards, an impact load is applied to the face guard, and the stress at the hinge point of the first-level face guard and the top beam or the telescopic beam is selected as the research object. The load of the coal wall on the two types of face guard is 45 $\mathrm{kN}$, and the loading position is the same as that shown in Fig. 9.

Fig. 11 shows the results of mechanical response characteristics of the hinged joints of the two types of face guard under coal wall caving. The comparison of Figs. $11 \mathrm{a}$ and $11 \mathrm{~b}$ shows that the stress at the hinge point of the integral face guard and the telescopic beam is generally smaller than that at the hinge point of the split face guard and the top beam. The average stress on the hinge points of the integral face guard is approximately $80 \%$ that of the split face guard. Stress on the hinge point of the integral face guard is only greater than that of the split face guard in a small area of $\mathrm{X}<-330 \mathrm{~mm}$, but the difference is relatively small. Therefore, compared with that at the integral face guard, the pin at the hinge point of the split face guard requires greater bearing capacity, and the stress condition is relatively bad. That is, the pin at the hinge of the split face guard is more prone to wear, breaking, and other failures.

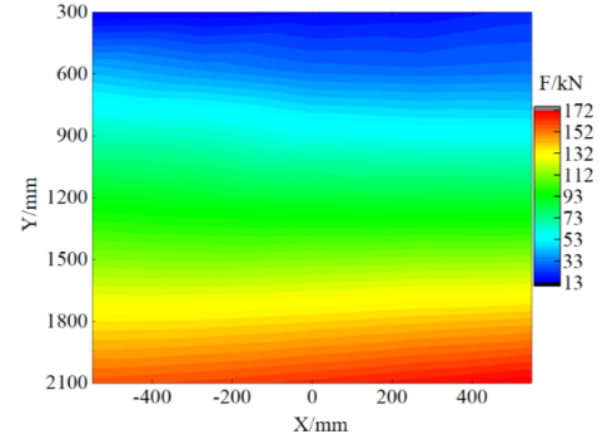

a) Integral face guard

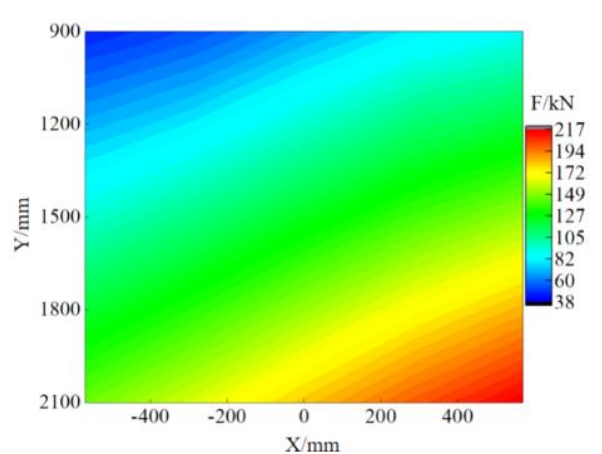

b) Split face guard

Figure 11: Mechanical response characteristics of the hinge joint of the face guard.

\subsection{Mechanical characteristics of the four-bar linkage with the integral face guard}

To study the mechanical characteristics of the other articulations of the four-bar linkage with the integral face guard by using the aforementioned numerical simulation model and the loading method presented in Section 4.2, an impact load of $45 \mathrm{kN}$ is applied to the face guard, and only the hinge point in the positive direction of the $\mathrm{X}$-axis is analysed.

In accordance with the result analysis shown in Fig. 12, the bearing characteristics of the hinge point of connecting rod $\mathrm{A}$ and the face guard, the bearing characteristics of the hinge point of connecting rods $\mathrm{A}$ and $\mathrm{B}$, and the bearing characteristics of the hinge point of connecting rod B and the telescopic beam (the same as the load-bearing characteristics of the hinge point of connecting rods $\mathrm{A}$ and $\mathrm{B}$ ) are affected by the state of the coal wall, and the change trend of stress is the same. When the value of the Y-axis is the same, stress at the 
hinge point increases with an increase in the $\mathrm{X}$-axis; when the value of the $\mathrm{X}$-axis is the same, stress at the hinge point increases with an increase in the $\mathrm{Y}$-axis.

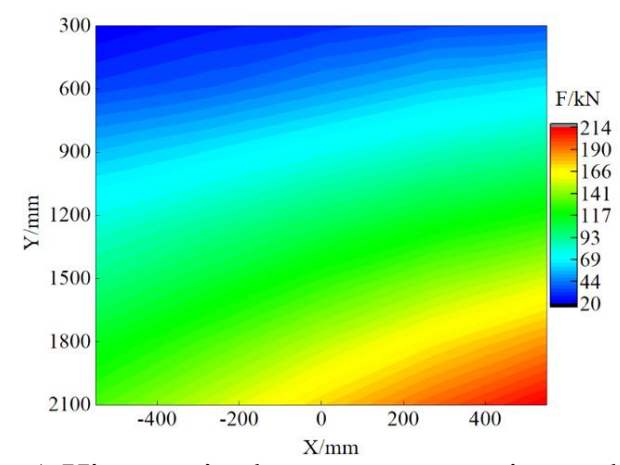

a) Hinge point between connecting rod $\mathrm{A}$ and the face guard

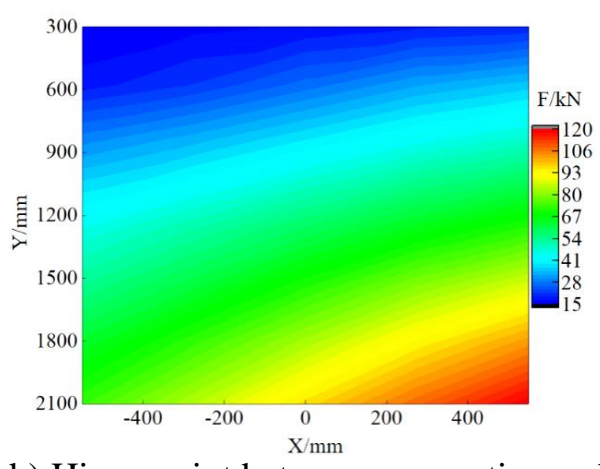

b) Hinge point between connecting rods $\mathrm{A}$ and $\mathrm{B}$

Figure 12: Mechanical response characteristics of the hinge joint of the four-bar linkage with the face guard.

On the basis of the data provided in Fig. 11 a, when the coal wall generates an impact load on the face guard under the integral face guard mechanism, the maximum stress at the hinge point of the face guard and the telescopic beam is $172 \mathrm{kN}$. The maximum stress at the hinge point between connecting rod $\mathrm{A}$ and the face guard is $214 \mathrm{kN}$. The maximum stress at the hinge point between connecting rods $\mathrm{A}$ and $\mathrm{B}$ and that at the hinge point between connecting rod $\mathrm{B}$ and the telescopic beam are $119 \mathrm{kN}$. Therefore, in designing and selecting materials for the hydraulic support of an integral face guard, priority should be given to meeting the use requirements of the pin shaft to avoid damage and failure under various working conditions because the limit stress of the pin shaft at connecting rod A and the face guard during use is larger than those at the other hinge points.

\subsection{Influence of coal wall angle on the stress of the face guard mechanisms}

The coal wall is not smooth, with various angles between the coal wall and the floor, due to other factors, such as shearer cutting and coal wall caving before face guard support. In accordance with the different conditions of the coal wall, hydraulic support should use its face guard mechanism to cooperate with the telescopic beam to make the position and posture of the face guard consistent with those of the coal wall, as shown in Fig. 13.

By applying the same numerical simulation method and in accordance with the effective liquid column length of the jack under different angles, the corresponding equivalent spring stiffness coefficient is calculated using Eq. (5), and the stress response characteristics of the retaining mechanism are analysed under different angles. Only the central position of the firstlevel face guard is loaded, and the results of the stress change of the hinge joint are shown in Fig. 14.

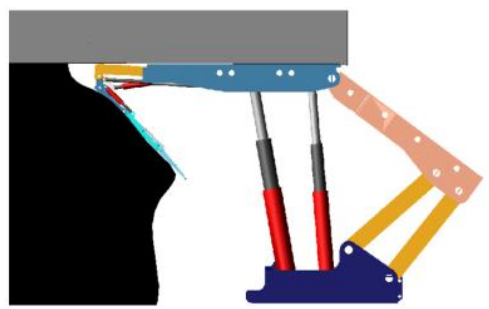

a) $67.5^{\circ}$ opening angle of the face guard

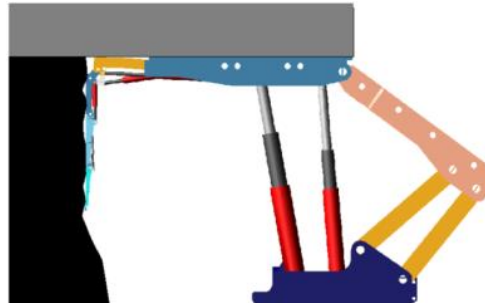

b) $90^{\circ}$ opening angle of the face guard

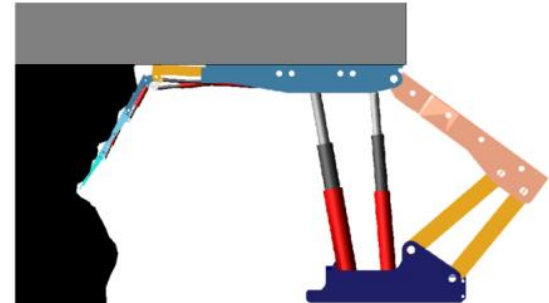

c) $112.5^{\circ}$ opening angle of the face guard

Figure 13: Different supporting angles of the integral face guard. 


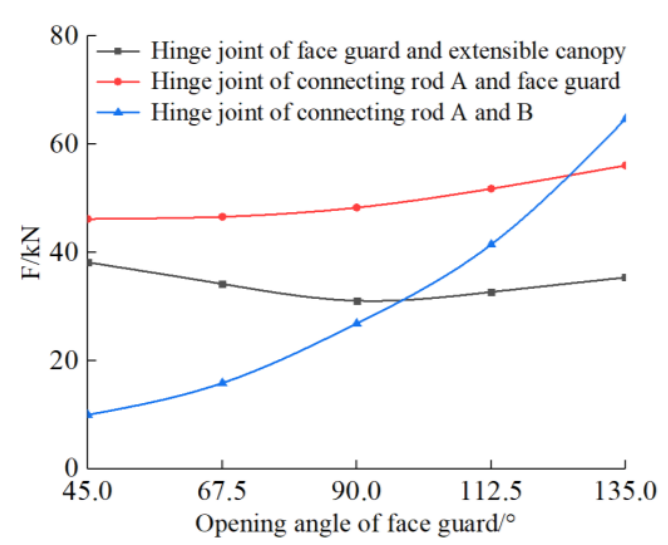

Figure 14: Effect of face guard angle on stress characteristics.

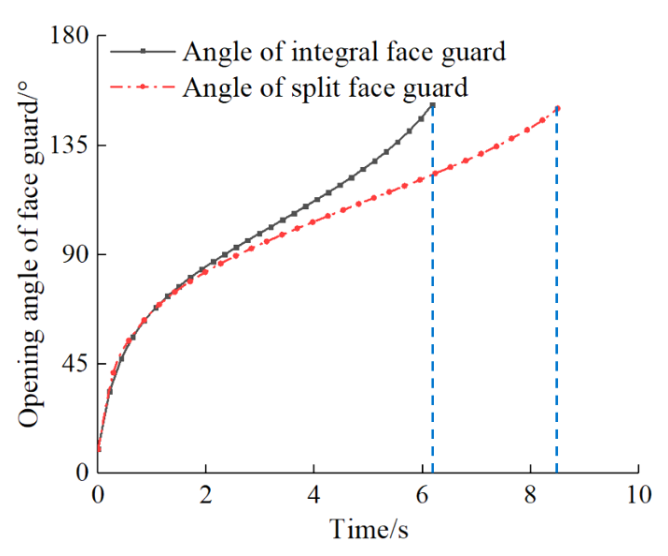

Figure 15: Angle change of the face guard mechanism.

As shown in Fig. 14, the influence of the opening angle of the face guard (i.e., the angle between the face guard and the top beam) on the hinge joint of the face guard and the telescopic beam and that of rod A and the face guard is relatively small. The maximum difference of stress change is approximately $10 \mathrm{kN}$. The hinge joint of connecting rods A and $\mathrm{B}$ is sensitive to the change in angle of the face guard, and stress increases gradually with an increase in the angle of the face guard. When the opening angle of the face guard is $135^{\circ}$, stress on the hinge point can reach up to $64.7 \mathrm{kN}$. Therefore, attention should be given to meeting the load-bearing conditions at the hinge joint of connecting rods $\mathrm{A}$ and $\mathrm{B}$ in the design and material selection of the hydraulic support's retaining mechanism.

\subsection{Stability analysis of the mechanism of the integral and split face guards}

To compare the influences of the integral and split face guard structures on the static stability of the coal wall, the current study determines the time required for the face guard to reach the expected supporting state to reflect the efficiency of changing the stress conditions of the coal wall, while simultaneously serving as the evaluation index of the face guard in maintaining the static stability of the coal wall.

In accordance with the numerical model presented in Subsection 3.2, measurement points are established at the front end of the second-level face guard to study the action efficiency of the two face guard mechanisms, and thus, reflect their effect on the static stability of the coal wall. The results are shown in Fig. 15.

Measurement begins when the horizontal angle between the two face guards and the top beam is set as $10^{\circ}$. When the face guard jack maintains the same and constant extension speed, the integral face guard takes only approximately $6.1 \mathrm{~s}$ to be stretched from the starting position to an angle of $150^{\circ}$ while the split type takes approximately $8.5 \mathrm{~s}$. Therefore, from the perspective of action efficiency and under the premise of the same disturbance on the coal wall, the integral face guard is conducive to maintaining the stability of the coal wall.

\section{CONCLUSIONS}

On the basis of the multi-angle investigation of integral and split face guards, this study obtains relevant conclusions regarding the stability of wall protection in a large mining height working face.

(1) In accordance with the different characteristics and formation mechanism of coal wall caving and after a comparative analysis of the action characteristics of face guards, various components should be coordinated to make the face guards come in contact with the coal wall at an angle of approximately $90^{\circ}$ and reduce the influence of dynamic disturbance on the coal 
wall. The integral face guard exhibits higher supporting efficiency than the split face guard. It can reduce the exposure time of the coal wall after shearer cutting, and it exhibits high flexibility and has a large supporting area. The integral face guard can also change the stress state of the coal wall more efficiently and reduce the occurrence of coal wall caving.

(2) Through theoretical calculations and numerical simulation analyses, the integral face guard is determined to be superior to the split face guard in terms of ultimate bearing capacity and supporting bearing range, and thus, it exhibits better coal wall support performance. The maximum stress at the hinge point of the integral face guard is lower than that at the hinge point of the split face guard; hence, the integral face guard achieves relatively higher reliability. The hinge pin of the integral face guard is less likely to be damaged with the same material.

(3) In the four-bar linkage mechanism of the integral face guard, the stress at the hinge point of connecting rod A and the face guard is larger. As the support angle of the face guard increases, stress at connecting rods $\mathrm{A}$ and $\mathrm{B}$ exhibits evident changes with a considerable increase. In the optimization design, the load-bearing conditions of the two pins should be considered first to avoid failure.

To study the contact characteristics of the face guard mechanism under different working conditions, double-stage variable stiffness is adopted during model establishment. This stiffness is slightly different from the real stiffness used in hydraulic support. In the future, continuously variable stiffness must be introduced into and considered for equivalent replacement during model establishment.

\section{ACKNOWLEDGEMENT}

This work was supported by the Coal Science and Industry Group Science and Technology Innovation Fund Project of China (Grant Nos. 2018-TD-ZD015 and 2020-TD-ZD015).

\section{REFERENCES}

[1] BP Amoco. Statistical Review of World Energy, from https://www.bp.com/en/global/corporate/ energy-economics/statistical-review-of-world-energy.html, accessed on 17-06-2020

[2] Mangal, A.; Paul, P. S. (2016). Rock mechanical investigation of strata loading characteristics to assess caving and requirement of support resistance in a mechanized powered support longwall face, International Journal of Mining Science and Technology, Vol. 26, No. 6, 1081-1087, doi:10.1016/j.ijmst.2016.09.017

[3] Xu, Y. X.; Wang, G. F.; Li, M. Z.; Zhang, J. H.; Han, H. J. (2019). Structure coupling between hydraulic roof support and surrounding rock in extra-thick and hard coal seam with super large cutting height and longwall top coal caving operation, Journal of the China Coal Society, Vol. 44, No. 6, 1666-1678, doi:10.13225/j.cnki.jccs.2019.0073

[4] Singh, G. S. P.; Singh, U. K. (2010). Prediction of caving behavior of strata and optimum rating of hydraulic powered support for longwall workings, International Journal of Rock Mechanics and Mining Sciences, Vol. 47, No. 1, 1-16, doi:10.1016/j.ijrmms.2009.09.001

[5] Yang, Z. K.; Sun, Z. Y.; Jiang, S. B.; Mao, Q. H.; Liu, P.; Xu, C. Z. (2020). Structural analysis on impact-mechanical properties of ultra-high hydraulic support, International Journal of Simulation Modelling, Vol. 19, No. 1, 17-28, doi:10.2507/IJSIMM19-1-498

[6] Li, X.; Liu, C.; Liu, Y.; Xie, H. (2017). The breaking span of thick and hard roof based on the thick plate theory and strain energy distribution characteristics of coal seam and its application, Mathematical Problems in Engineering, Vol. 2017, Paper 3629156, 14 pages, doi:10.1155/ 2017/3629156

[7] Cheng, J. Y.; Wan, Z. J.; Li, W. F.; Ji, Y. L.; Wang, S. H. (2015). A comprehensive methodology for predicting shield support hazards for a U.S. coal mine, Dyna-Ingenieria e Industria, Vol. 90, No. 4, 442-450, doi: $\underline{10.6036 / 7580}$ 
[8] Pang, Y. H.; Wang, G. F.; Ren, H. W. (2019). Multiple influence factor sensitivity analysis on coal wall spalling of workface with large mining height, Journal of Mining and Safety Engineering, Vol. 36, No. 4, 736-745, doi:10.13545/j.cnki.jmse.2019.04.012

[9] Liu, Q. (2019). Study on the Control of Rib Fall of Coal Wall Based on the Working State of Large Mining Hydraulic Support, Master Thesis, Taiyuan University of Technology, Taiyuan (in Chinese)

[10] Ge, X.; Xie, J. C.; Wang, X. W.; Liu, Y.; Shi, H. B. (2020). A virtual adjustment method and experimental study of the support attitude of hydraulic support groups in propulsion state, Measurement, Vol. 158, Paper 107743, 13 pages, doi:10.1016/j.measurement.2020.107743

[11] Delic, M.; Mandic, V.; Popovic, M. (2021). Stability analysis of steel welded tubes forming process using numerical simulations, Tehnicki glasnik - Technical Journal, Vol. 15, No. 2, 298304, doi:10.31803/tg-20210504112813

[12] Hu, X. P. (2020). Cooperative automatic control for the canopy posture of a four-leg hydraulic support, International Journal of Simulation Modelling, Vol. 19, No. 4, 713-724, doi:10.2507/IJSIMM19-4-CO20

[13] Behera, B.; Yadav, A.; Singh, G. S. P.; Sharma, S. K. (2020). A numerical modeling approach for evaluation of spalling associated face instability in longwall workings under massive sandstone roof, Engineering Failure Analysis, Vol. 117, Paper 104927, 29 pages, doi:10.1016/ j.engfailanal.2020.104927

[14] Verma, A. K.; Deb, D. (2013). Numerical analysis of an interaction between hydraulic-powered support and surrounding rock strata, International Journal of Geomechanics, Vol. 13, No. 2, 181 192, doi:10.1061/(ASCE)GM.1943-5622.0000190

[15] Zeng, X. T.; Meng, G. Y.; Zhou, J. H. (2018). Analysis on the pose and dynamic response of hydraulic support under dual impact loads, International Journal of Simulation Modelling, Vol. 17, No. 1, 69-80, doi:10.2507/IJSIMM17(1)412

[16] Witek, M.; Prusek, S. (2016). Numerical calculations of shield support stress based on laboratory test results, Computers and Geotechnics, Vol. 72, 74-88, doi:10.1016/j.compgeo.2015.11.007

[17] Liang, L. C.; Ren, H. W.; Zheng, H. (2018). Analysis on mechanical-hydraulic coupling rigidity characteristics of hydraulic powered support, Coal Science and Technology, Vol. 46, No. 3, 141147, doi:10.13199/j.cnki.cst.2018.03.024

[18] Islavath, S. R.; Deb, D.; Kumar, H. (2016). Numerical analysis of a longwall mining cycle and development of a composite longwall index, International Journal of Rock Mechanics and Mining Sciences, Vol. 89, 43-54, doi:10.1016/j.ijrmms.2016.08.003

[19] Liu, S.; Yang, K.; Zhang, T.; Tang, C. N. (2020). Rib spalling 3D model for soft coal seam faces with large mining height in protective seam mining: theoretical and numerical analyses, Geofluids, Vol. 2020, Paper 8828844, 17 pages, doi:10.1155/2020/8828844

[20] Wan, L. R.; Zhang, S.; Meng, Z. S.; Xie, Y. Y. (2021). Analysis of the protection performance of face guard for large mining height hydraulic support, Shock and Vibration, Vol. 2021, Paper 6631017, 16 pages, doi:10.1155/2021/6631017

[21] Meng, Z. S.; Zeng, Q. L.; Gao, K. D.; Kong, S.; Liu, P.; Wan, L. R. (2018). Failure analysis of super-large mining height powered support, Engineering Failure Analysis, Vol. 92, 378-391, doi:10.1016/j.engfailanal.2018.04.011 